\title{
CECIL CRAWFORD O'GORMAN
}

$1874-1943$

\author{
POR \\ JUSTINO FERNANDEZ
}

I A vida de Cecil Crawford O'Gorman vale tanto como su obra o viceversa, porque en ambas se encuentra el mismo tono de aventura y distinción. Como hombre del norte buscó el clima templado para vivir y México le proporcionó el lugar donde desarrollar su espiritu. Aunque vino joven ya traía formación e impresiones de otros sitios; nacido en Inglaterra, de padres irlandeses, educado por los benedictinos, había crecido al lado de una tía suya, mujer refinada, conocedora en arte y pintora ella misma de acuarelas que recibieron la mejor acogida en su tiempo; con clla visitó Mr. Cecil los museos y allí empezó su cavilar en el arte que no abandonó en la vida entera. Pasó largas temporadas en Cannes y por fin se embarcó para la aventura americana a los veintiún años, en 1895.

En México encontró la rama mexicana de su familia, cuyo origen en América se remonta al primer cuarto del siglo xix, cuando vino el primer O'Gorman, don Carlos, en la misión en que figuraban Ward y Harvey. Casó a los treinta años con su prima en segundo grado doña Encarnación 
O'Gorman y Moreno y se estableció como ingeniero de minas; Coyoacán, entonces pueblo apacible fué su lugar de residencia, más tarde Guanajuato $y$, por último, se instaló en San Angel, en una vieja casona, donde pudo ir arreglando con sus propias manos, distribuyendo aqui, pintando allá, objetos antiguos del México virreinal que con sumo tino $y$ antes de que fueran apreciados había ido reuniendo. Esa casa del Callejón del Santísimo, ahora número 6 , hay que contarla entre las obras del artista porque refleja, como sus pinturas, su personalidad. Cuatro hijos tuvo el matrimonio que temprano mostraron sus aficiones: Juan por la pintura y la arquitectura, Edmundo por las letras, Margarita (hoy Mrs. Von Waberer) por la buena conversación y Tomás por el sport y más tarde por la música.

En aquella casa llena de encanto, con su patio y corredor invadido por plantas trepadoras, con su fuente de viejos azulejos, con sus cuartos inundados de tallas coloniales, de pinturas, caí un buen dia como esas visitas de "week end", que se quedan para el resto de la vida. Para un aficionado a la pintura, a las letras y a la conversación como era yo, aquella casa era Jauja. Mr. Cecil pintaba día y noche, comentábamos y discutiamos sus pinturas; Juan revolucionaba la arquitectura deslumbrándonos con sus ideas funcionalistas; Edmundo siempre con alguna nueva teoria en la cabeza, dirigía las conversaciones que Margarita remataba con golpes de ingenio y yo, intentaba, cariñosamente, seguir los intereses individuales, en interés propio. Por aquella casa, domingo a domingo, fué desfilando cuanta personalidad interesante había en México y cuantas venían del extranjero; pronto apareció también uno que otro turista, que si bien no alternaba en la conversación sí lo hacía en los ricos platos preparados por la virtuosa señora $O^{\prime}$ Gorman o, como la llamamos familiarmente. Chonita. En ese ambiente Mr. Cecil pintó la mayor parte y lo mejor de su obra, alli vivió el resto de su vida, salvo una que otra escapatoria a los Estados Unidos o a Europa. Las exposiciones de sus cuadros se sucedieron desde 1915 hasta 1933, ya en México, ya en el extranjero, ya en su propia casa que hacía un marco espléndido para las pinturas. Tras los años de la Revolución, de donde data un documento curioso, fímado por el C. Primer Jefe don Venustiano Carranza, en que se nombra a $\mathrm{Mr}$. Cecil inspector honorario de Monumentos Artísticos, por su gusto y conocimiento y por haber salvado en más de una ocasión del vandalismo algunos monumentos de arte, pasa a Nueva York en 1917, en los momentos en que la pintura moderna estaba en auge. Mr. Cecil result 6 novedoso porque sus obras sinceras y refinadas no se parecian a lo que podia verse en las galerias de la calle 57; el éxito fué rotundo, no sólo vendió todo lo que 
expuso, sino que recibió los más grandes elogios. Dos años más tarde exhibe en San Antonio y nuevamente en Nueva York con igual éxito y en los años siguientes en su casa de San Angel, se reunían periódicamente conocedores y público selecto para ver las novedades que su inquieto espíritu iba produciendo. Fué una vez más a Europa, por corto tiempo, una vez más tuvo el ánimo suficiente para ir a California, pero en los últimos diez años de su vida, ni viajó ni expuso más y fueron, quizá, los más fecundos.

El paisaje mexicano le atrajo siempre, así como las ruinas, los conventos, las iglesias del pasado colonial, todo lo pintó con extrema finura, con delicado sentimiento y certero color; fué un hábil impresionista, como correspondía a su amor por la naturaleza y a sus aficiones científicas, pero nunca confundió esas inclinaciones con la creación artística. Experto en la acuarela, que practicó desde joven, logró espléndidas obras, de una diafanidad y una elegencia auténtica, excentas de teatralidad. Las nuevas corrientes de la pintura fueron motivo de muy sabrosas especulaciones y con penetrante mirada supo distinguir lo bueno de lo malo, jamás le dieron gato por liebre, pero su fino espíritu vió invadir el mundo de vulgaridades que no podía aceptar, supo guardar el equilibrio a través de todos los vaivenes, quizá lo guardó demasiado y el resultạdo fué que sólo asimiló aquello que encontró más razonable. Tuvo el tino de no perder idea de sus limitaciones, la inteligencia de resumir sus conocimientos en la etapa final de su obra sin intentar grandes novedades y el control necesario para no caer en lo falsamente novedoso; en verdad ese plantarse en lo que era, en lo que sabía hacer y en hacerlo de la mejor manera posible fué su última gran aventura, y le salió bien; se jugó su última carta con valor, con honradez, con sinceridad, no permitió que lo engañasen los cantos de la sirenas (en este caso mecánicas) y con clarividencia de los años que aun le quedaban por delante, se puso a pintar retratos llevando al límite sus facultades, desdeñando el exhibicionismo y la adulación y quizá desdeñando también el mundo de horror $y$ de vulgaridad por el que transcurren nuestras vidas.

Así pasó sus últimos años; alto, delgado, siempre cordial, con aristocrática y genuina naturalidad dispuesto en todo momento a leer un buen libro, de literatura o de ciencia, y a entablar una conversación. Su ascetismo lo llevó a recluirse en su estudio, sin lujos, sin afectación, con un sillón viejo, una taza de té a la mano y fumando incesantemente frente al caballete que sostenía su obra en plan de realización. Así pasamos juntos muchas tardes, muchas horas, charlando sobre arte y sobre mil cosas 
más, pero su tema favorito era el científico ante el cual me inclinaba con vacilación y escuchaba con respeto, de él aprendí algo en este aspecto, tuve que leer a Eddington y a James y por fin caí en cuenta de que $\mathrm{Mr}$. Cecil, mejor que por ninguna otra vía, por la cientifica, había encontrado un nuevo sentido a la vida, comprendido el de nuestra época y vislumbrado toda una era futura. Einstein era el objeto de su mayor admiración. De alguna de nuestras conversaciones nació la idea de que escribiese el artículo que se publicó en los Anales del Instituto de Investigaciones Estéticas, bajo el título de The Painter lays down his brushes and turns critic. (No 4, 1939.) En ese artículo Mr. Cecil se dice a si mismo: "It seems time to ask myself whether my work is artistic. I do not know why I should bother about this at all, but $I$ confess to a vain but legitimate desire to live in my work after I am dead..." ; intentó resumir sus ideas que expresó con claridad, añadiendo: "I am not setting out to challenge any philosophy, nor have I any desire to suggest a new theory about the Universe"; su conocimiento del arte moderno era extenso y cabal: "It enables the artist to produce nature forms without the somewhat childish necessity of direct imitation. The best modernistic pictures arrive at this quality in a clean direct manner which gives them a high decorative value." Las formas naturales sin necesidad de imitación y la calidad decorativa, fueron la base de la pintura de Mr. Cecil y, por último, tras de su dìsertación para distinguir entre Arte y Belleza, nos da su concepto del primero: "Art considered in this brood sense is man's attempt to fulfill an inborn desire, whether in verse, stone, paint, or music, to attain the infinite and explain the longings which are part of his heritage."

Cómo expresó Mr. Cecil la interpretación de las formas naturales, dentro de un sentido decorativo, ha de encontrarse sobre todo en sus ultimos retratos, a los cuales dedicó don Manuel Toussaint un estudio $R \boldsymbol{e}$ trato y paisaje en la obra de Cecil Crawerord O'Gorman ("Alcancia". México, 1938). El artista terminó con empeño su última obra y tranquilamente pasó a mejor vida.

Con su reciente muerte (23 de noviembre de 1943) acaecida cuando lograba sus mejores triunfos, hemos perdido uno de los mejores retratistas que hayan florecido en nuestro medio y, a la vez, a un hombre probo y refinado, cuyo sentido de la vida puede resumirse en un positivo horror a la vulgaridad.

En enero de 1944, la Dirección General de Educación Estética organizó una exposición en homenaje a la memoria del pintor, que tuvo lugar en el Palacio de Bellas Artes; allí pudo apreciarse el valor de su obra, en 


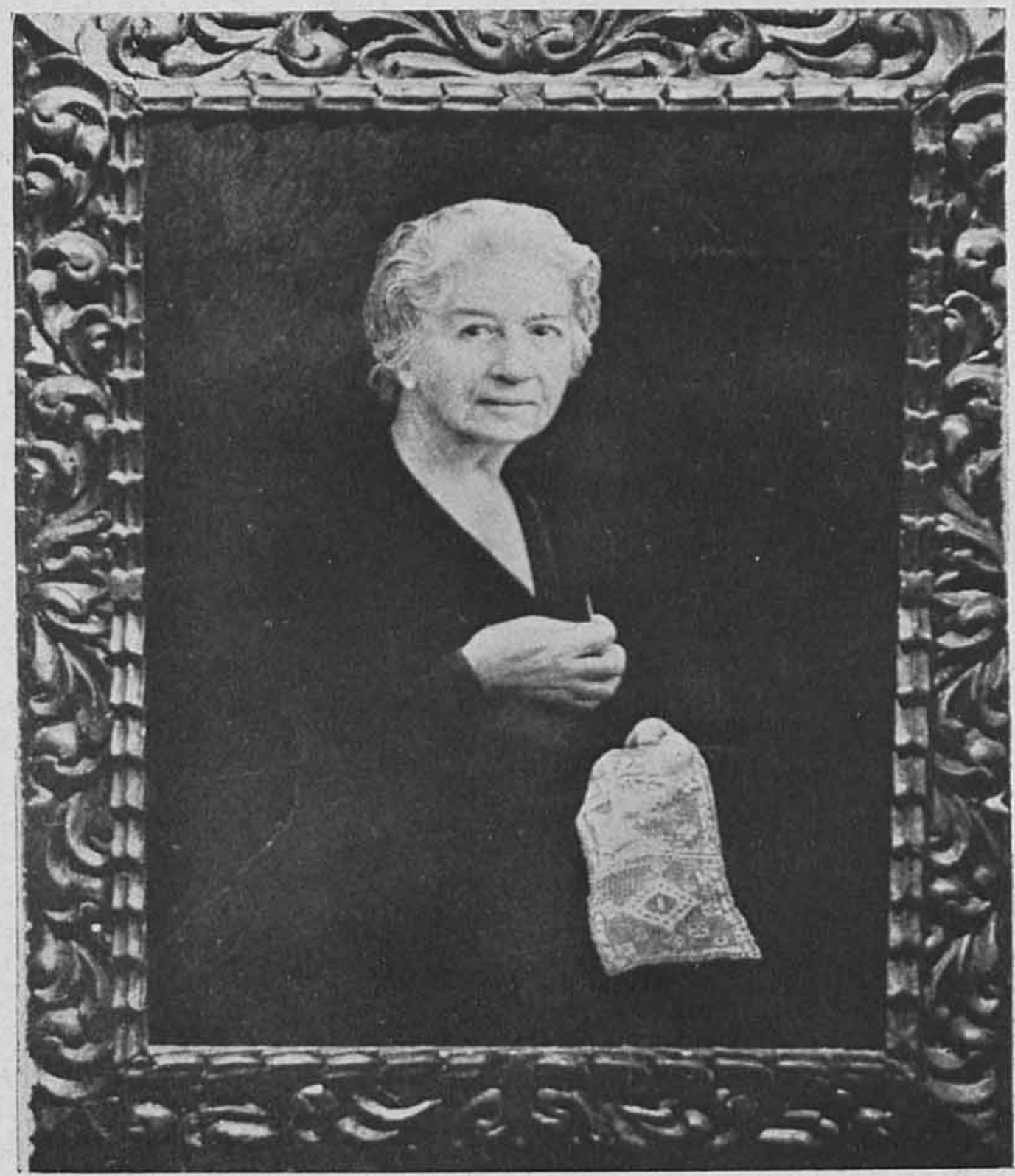

1. Señora Encarnación $O^{\prime} G$. Vda. de $O^{\prime}$ Ǵorman. 
DOI: http://dx.doi.org/10.22201/iie.18703062e.1944.11.368

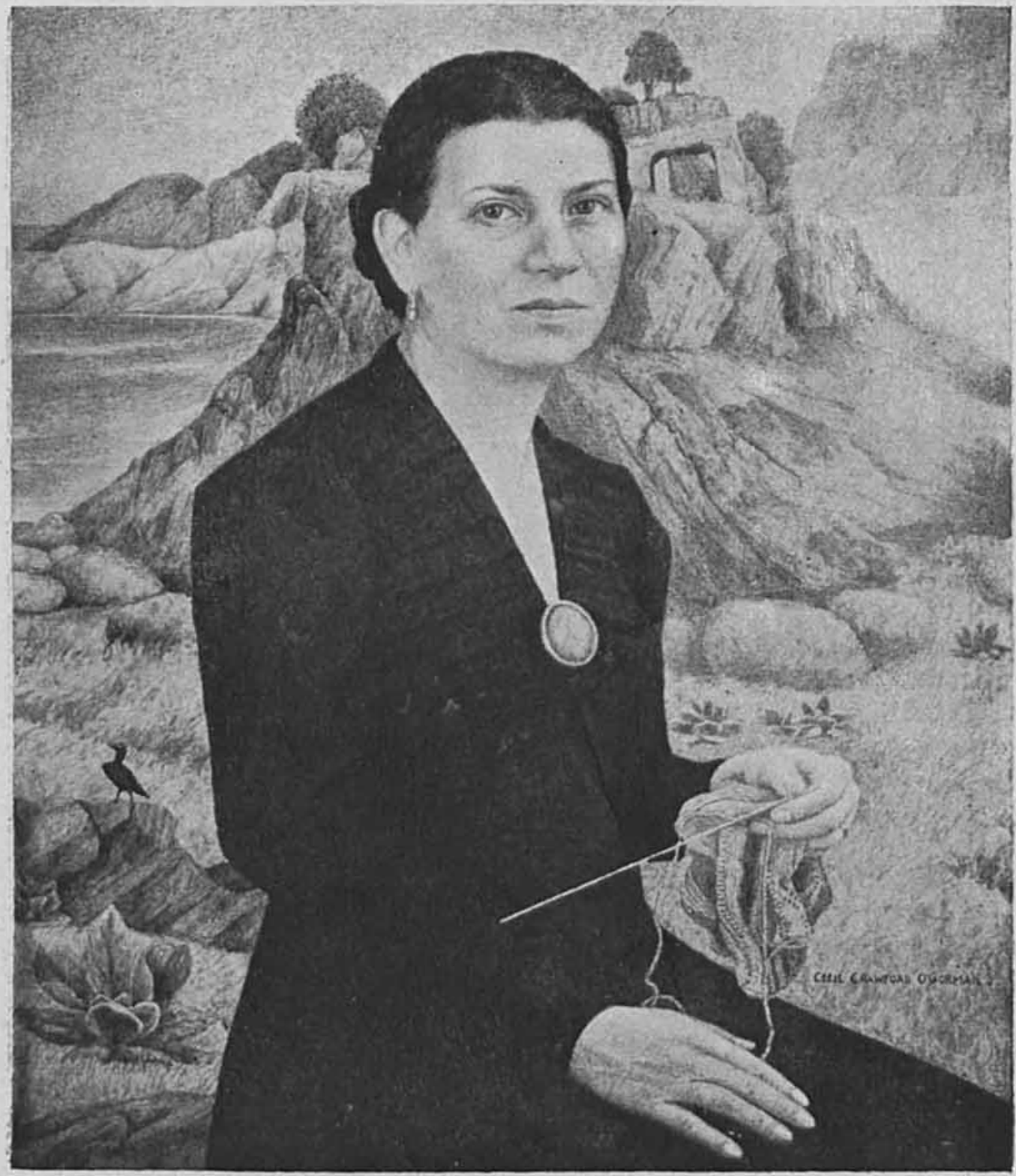

2. Señora Guadalupe Fernández del Valle de Cardona. 
unos cuantos, escogidos cuadros. La excelencia de su técnica, muestra de un amor al oficio, es, a mi modo de ver, una lección ejemplar; trabajó sobre tablas preparadas por él mismo, pintando con acuarela, por transparencias, tocando al final con óleo y encerando la superficie total; a este medio, a la vez arduo y delicado, que requiere sabiduría y precisión, le debió quizá sus mejores producciones, cuando poseedor de rica experiencia, lo único que le interesó fué lograr el máximo de sus posibilidades, consciente de sus propias limitaciones.

Sus cualidades básicas fueron un fino y certero sentido del color, una aptitud excepcional para la composición y una calidad decorativa que fué elemento "sine qua non" de sus obras. De las expuestas en Bellas Artes quiero destacar tan sólo dọ de ellas que resumen sus tendencias. Una de ellas es el retrato de la señora O'Gorman (1940) que aparece sentada, vestida de negro, con una costura entre las manos; la severidad fué una de las exigencias del artista en sus retratos, unida a una amable disposición, evitando el violento dramatismo, ambas cualidades aparecen en el retrato que nos ocupa, pero lo mejor en él es la cabeza de la figura de un preciso y precioso realismo, en el sentido cabal de la palabra, pues no sólo el aspecto físico está representado sino que va unido a una interpretación psicológica que verdaderamente anima el rostro y da vida a la mirada; el cabello sabiamente estilizado no deja duda de que no se trata de una imitación naturalista, sino de una obra de arte de extraordinaria sintesis; por lo delicado de la factura y la fina observación del modelo, este retrato es uno de los mejores que se han producido en nuestro medio en los últimos años.

El otro retrato que me parece también excepcional, es el de la señora Guadalupe Fernández del Valle de Cardona (1941), distinto del anterior, por más de un concepto. Ya hemos visto que el paisaje y el retrato fueron los dos temas fundamentales en la obra de Mr. Cecil; en el primero recorrió un camino que va desde el naturalista hasta el irnaginativo, pasando por muchas otras formas de interpretación, al segundo llegó por convicción, por tener aguda conciencia de lo superfluo del arte en nuestro tiempo y encontró en él la razón de seguir pintando, por sentir que, a lo menos, el tema tenía sentido, evitando la banalidad. Cuando quiso resumir sus conocimientos fué a dar precisamente en la síntesis de sus dos temas, el paisaje y el retrato, $y$ así logró una perfecta unidad en la obra que analizó y que, a mi modo de ver, es la mejor de cuantas pintó y un verdadero clímax de su obra. En ningún otro retrato como en éste la figura encaja en el paisaje en armonía absoluta, la unidad de la expresión y del tratamiento es patente, tanto en la figura vestida de negro, como en el paisaje 
imaginativo que le sirve de fondo, formado por rocas y vegetales; la interpretación de las líneas del rostro, de exquisito y preciso dibujo, sin llegar jamás a la imitación vil; los recuerdos lejanos de su familiaridad con las obras de los "primitivos" italianos y flamencos; el humorismo aparente en aquel pajarraco negro que surge en el paisaje, sin atraer demasiado la atención y la calavera, casi imperceptible, también en el fondo, pone un toque de realismo extremo; el colorido, unificado, rico y finamente contrastado, por la figura de negro sobre el claro paisaje completa la obra que resume, por así decirlo, todo lo que el pintor sabía, todo lo que podía hacer, todo lo que era. Si Mr. Cecil no hubiese dejado otras obras bastaria ésta para reputarlo como un artista que supo lograr el punto de equilibrio entre el mundo objetivo y la imaginación creadora.

Las palabras del pintor confesando un vario, pero legítimo, deseo de vivir en su obra después de muerto, cobran ahora sentido; y su duda ". . and I know that tunless the work is considered artistic, I stand but a small chance of a permanent place with future generations", queda, en lo que a mi se refiere, desechada, porque su obra es de arte puro y sin mácula y los que vengan detrás, si desean una lección de lo que el arte en todo tiempo es, pueden verla y estudiarla como ejemplo vivo de lo que es posible lograr cuando se es artista genuino. 